\title{
The Synchronous Optimization Design of Fixture Layout and Clamping Force based on UG
}

\author{
Junhua Zhao \\ Henan Polytechnic, Zhengzhou 450046, China \\ junhua_zhao@yeah.net
}

Keywords: Multi objective function, Fixture optimization, Genetic algorithm, Simulation, Adaptive, Finite element.

\begin{abstract}
In order to reduce the workpiece deformation in the machining process and improve the machining accuracy, this paper puts forward multi-objective fixture optimization method based on the objective function of workpiece even deformation and the minimizing machining deformation. Based on the optimized fixture layout, the method divides the processing path into a plurality of conditions and each condition uses genetic algorithm and finite element optimization algorithm to solve the optimal clamping force. In order to verify the effectiveness and the reliability of the algorithm, based on the ANSYS secondary development, this paper uses APDL to compile finite element solving model of parametric workpiece-fixture system, finally uses MATLAB language to compile the genetic algorithm program, doing simulation on the simultaneous optimization of fixture layout and the clamping force. Through the simulation calculation, this paper gets adaptive optimization curve of fixture layout and clamping force, which realizes simultaneous optimization of fixture layout and clamping force. It is an important improvement of fixture optimization method, which can greatly improve the performance of the fixture.
\end{abstract}

\section{Introduction}

In mechanical processing, the size of the parts, geometry and surface relative position are up to the mutual position relationship between the workpiece and tool in the cutting process. Fixture is the key link of machine tool, the tool and the workpiece, and fixture ensures machining, improves quality, increases productivity and expands the scope of the processing machine tool [1, 2]. Machine tool fixture comprises a positioning element, clamping element, the auxiliary positioning component, a connecting element and other elements [3, 4]. This paper uses ANSYS and MATLAB software to do simulation on the optimization layout of the fixture. The overall design framework is as follows:

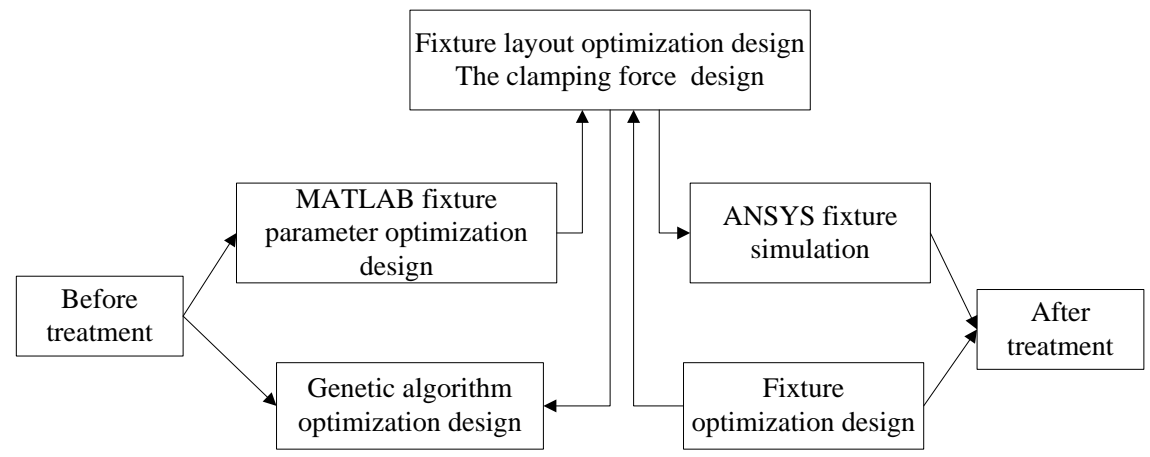

Fig.1: Design framework of fixture layout optimization

From Figure 1 it can be seen, the fixture layout optimization design includes optimization design of clamping force and the overall layout. The optimization parameter uses genetic algorithm to carry on the code and uses MATLAB software to program [5]. The optimized parameter uses the ANSYS software to do simulation. The post processing results are analyzed, so as to realize the optimization design of fixture. 


\section{The Mathematical Model and Algorithm of Fixture Layout Optimization Design}

In the layout optimization design of fixture, the circle can use ABCDEF polygon approximation to replace, slot uses GHIJK to replace. In the process of fixture layout optimization, these points need to be stored, and the location of point needs to be coded, so it can use the objective function to optimize [6-8]. The fixture layout is as shown in Figure 2.

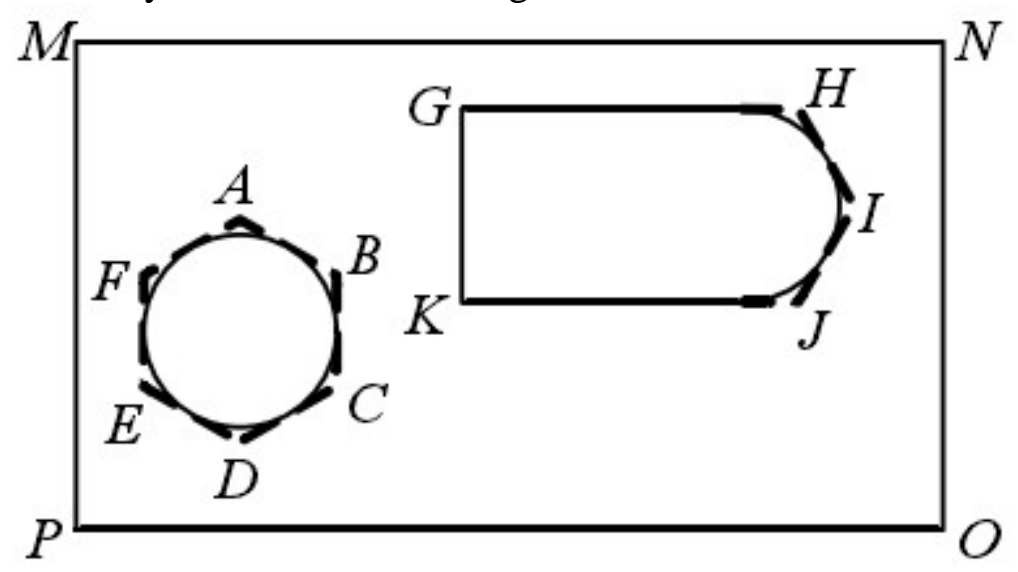

Fig.2: The sample positioning surface of machining

Based on the genetic algorithm, it will generate a random genetic sequence of clamping force.

$$
\left\{F^{0}{ }_{t-1}, F_{t-1}^{1}, F_{t-1}^{2}, \cdots, F_{t-1}^{n}\right\}, F^{0}{ }_{t-1} \in R .
$$

Among them, $F^{0}$ is initial clamping force, $F$ is clamping force. Multi objective function is consisting of different functions. It can use multi-objective optimization function to optimize clamping force, and its expression is as follows:

$$
\max \left\{\begin{array}{ccc}
a_{1}= & F_{11} w_{1}+F_{12} w_{2}+\cdots+F_{1 n} w_{n} \\
a_{2}= & F_{21} w_{1}+F_{22} w_{2}+\cdots+F_{2 n} w_{n} \\
\vdots & \vdots \\
a_{n}= & F_{n 1} w_{1}+F_{n 2} w_{2}+\cdots F_{n n} w_{n}
\end{array} .\right.
$$

Among them, $a$ is optimization value, $w$ is optimization coefficient. Clamping force is partition according to the path, as shown in the formula (3).

$$
F_{p}=\sqrt{\left(\frac{d^{2} F_{x}}{d S^{2}}\right)^{2}+\left(\frac{d^{2} F_{y}}{d S^{2}}\right)^{2}+\left(\frac{d^{2} F_{z}}{d S^{2}}\right)^{2}} .
$$

$F_{p}$ is optimized clamping force, $S$ is fixture layout area. So the finite element optimization goal of fixture stress is as follows:

$$
\sigma_{l}=\frac{1}{S}\left[F_{p}\left(\sum_{j=1}^{n} F_{j} w_{j}+F_{p} F_{j} w_{j}\right) a_{j}\right] .
$$

Among them, $\sigma_{l}$ shows fixture stress. In order to realize the simulation of fixture optimization design, this paper uses APDL parametric language to develop the simulation model of fixture, and the program is as follows:

*SET, length, 0.15

*SET, width, 0.1

*SET, height, 0.01

*SET, k1, 2.5323e7

*SET, k2, 3.1282e7

*SET, em1, 7.1e10

*SET, em2, 2.2E11 


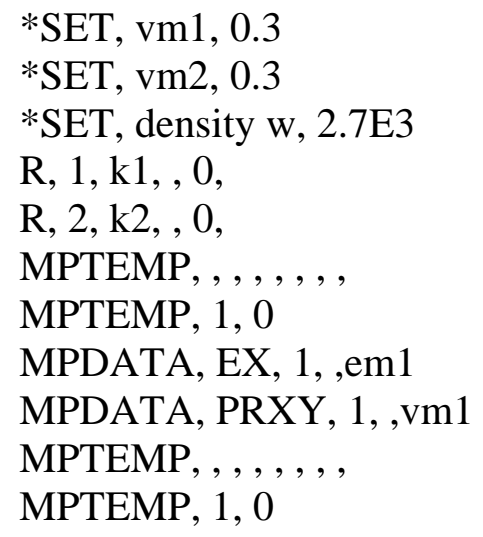

In order to optimize the clamping force and layout size of fixture, this paper uses genetic algorithm to calculate the fitness of the fixture and through iterative calculation method to reduce the convergence error. This paper uses MATLAB programming to design the algorithm, and the basic programming is as follows:

Begin

$\mathrm{t} \leftarrow 0$;

Initialization $\mathrm{F}(\mathrm{t})$;

Assessment $\mathrm{F}(\mathrm{t})$;

While don't meet the condition of termination do

Begin

Recombination $\mathrm{F}(\mathrm{t})$ can get $\mathrm{l}(\mathrm{t})$;

Assessment $\mathrm{l}(\mathrm{t})$;

From $\mathrm{F}(\mathrm{t})$ and $\mathrm{l}(\mathrm{t})$ select $\mathrm{F}(\mathrm{t}+1)$;

$\mathrm{t} \leftarrow \mathrm{t}+1$;

End

End

\section{Simulation of Fixture Clamping Force and Optimization Layout}

In order to effectively verify the effectiveness and reliability of fixture clamping force optimization layout designed in second section, this study adopts ANSYS software to do simulation on clamping force and optimization layout, and uses the multi-objective genetic algorithm to optimize the model [9-11]. The fixture layout is as shown in Figure 3.

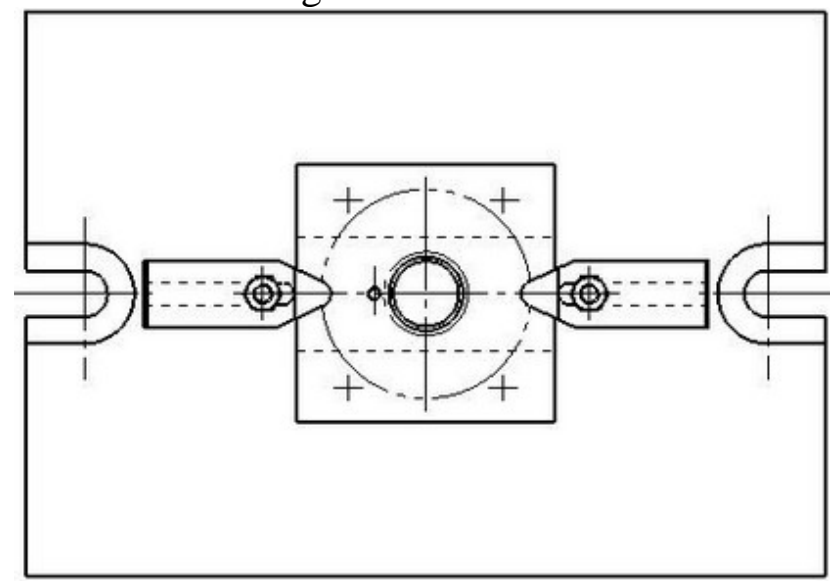

Fig.3: Schematic diagram of the fixture layout

Figure 3 shows the schematic diagram of the fixture layout. The fixture optimization layout uses thin component as the workpiece, and the hole is simplified into a polygon [12]. It uses the finite element software to modeling the workpiece, as shown in Figure 4. 


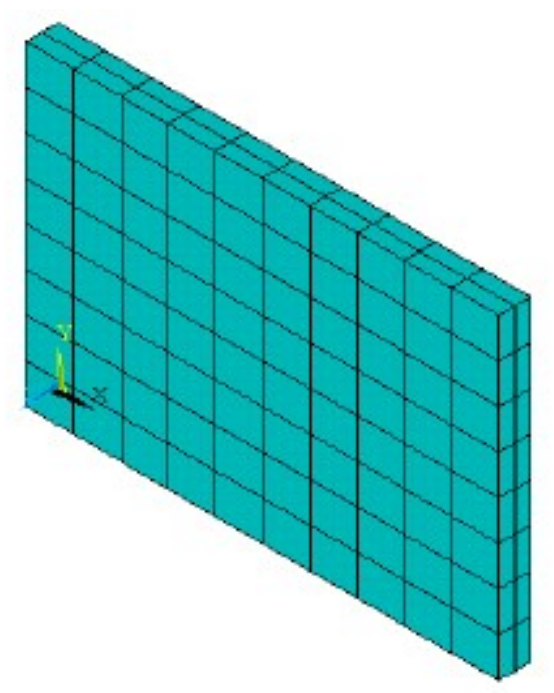

Fig.4: Finite element modeling of workpiece

As shown in Figure 4 it is the finite element model of workpiece. This paper adopts the bottomup modeling methods. In the solid model structure, it firstly defines the key points, and then uses these key points to define higher entity, such as the line and surface, finally generates body [12]. The body is done grid division, and the boundary conditions are set as shown in Figure 5.

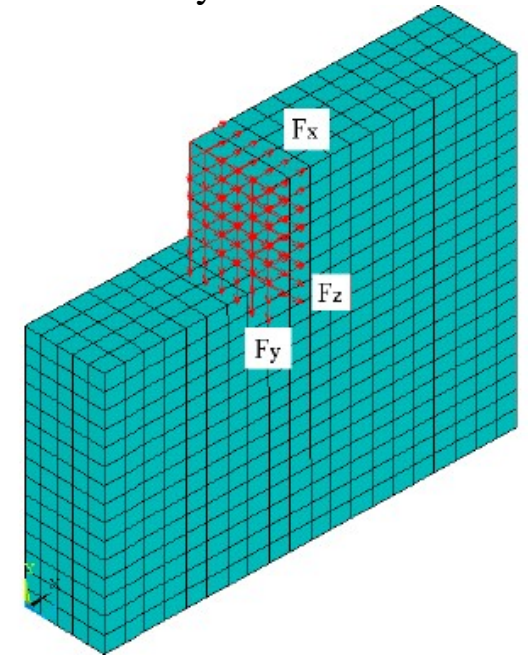

Fig.5: The simulation boundary conditions set

Figure 5 represents the boundary conditions set of workpiece clamping force. According to the fixture component position information, it can find the finite node around the contact points [13]. It use node selecting function of ANSYS to select a given range node, and applies the clamping force boundary conditions. The fitness calculation is as shown in Figure 6.

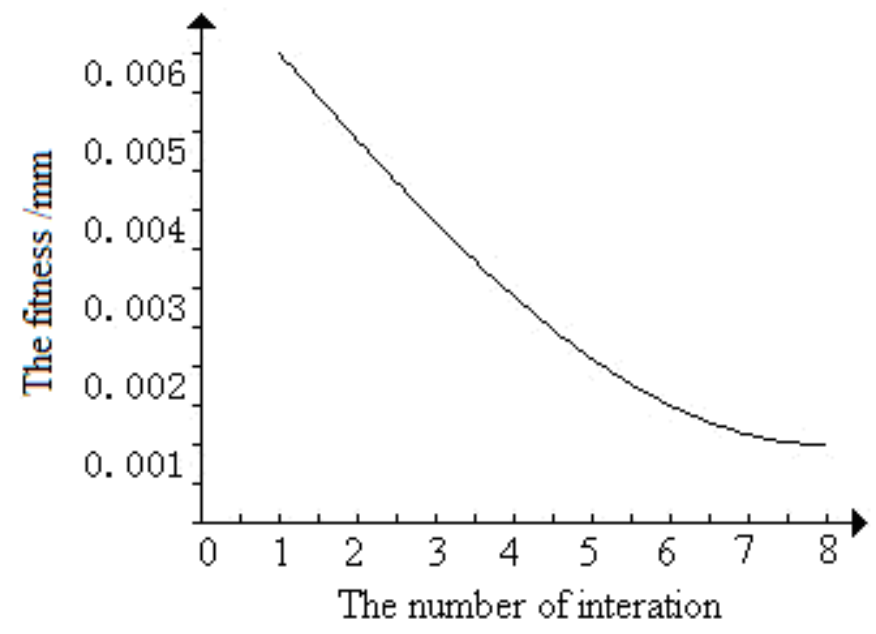

Fig.6: The calculation results of fitness iteration 
The convergence process of workpiece fixture layout and clamping force optimization is as shown in Figure 6. This paper uses multi objective genetic algorithm to optimize the layout of the fixture [14]. Through iterative calculation, fitness value is gradually reduced, and the final calculation results are smaller than the workpiece experience deformation, which achieves the purpose of optimization.

Table 1: Fixture layout and clamping force optimization table

\begin{tabular}{llll}
\hline & $\mathrm{X} / \mathrm{mm}$ & $\mathrm{Y} / \mathrm{mm}$ & $\mathrm{Z} / \mathrm{mm}$ \\
\hline L1 & 50 & 30.321 & 20.231 \\
L2 & 50 & 60.385 & 25.258 \\
L3 & 21.234 & 0 & 11.247 \\
F1 & 0 & 2985.132 & 0 \\
F2 & 0 & 3123.259 & 0 \\
F3 & 0 & 4002.239 & 0 \\
\hline
\end{tabular}

Through optimization parameters of Table 1, this paper uses finite element software ANSYS to simulate the layout, and the maximum workpiece deformation is $0.5524 \mathrm{~mm}$. Compared with the experience design, the maximum deformation value of optimization method is reduced by $52.3 \%$, and the required maximum clamping force is smaller than the experience design clamping force reduced by $25.2 \%$, which greatly improves the performance of the fixture.

\section{Summary}

In order to improve the machining precision of workpieces and reduce the workpiece deformation in milling process, this paper puts forward a new fixture layout optimization method. This method uses genetic algorithm to encode the clamping force and layout, and uses multiple objective function to optimize the parameters, finally uses the MATLAB software to design programming. This paper uses ANSYS software to do simulation on the fixture layout optimization, and uses MATLABT to obtain the optimized parameters in the simulation process. Through the simulation, optimization results of fixture optimization layout and clamping force are obtained, which provides a theoretical basis for optimization design of fixture performance.

\section{Acknowledgments}

The work was supported by the twelfth-five-year national science and technology support program Application Demonstration and Development of Three Dimensional Animation Technology with the program number 2009SJGLX339.And the program name is Application Research on Germany Mode of Action Oriented Vocational Education in the Teaching Practice of NC machining in in Higher Vocational College.

\section{References}

[1] J.H. Zhou, Q.W. Wang, J.M. Gao, M.S. Ma, X. Li. Gyroscope frame clamping deformation analysis based on finite element method. New technology, 2014, 2(1): 32-35.

[2] B.Z. Li i, J.G. Zhao, J.G. Yang, G.M. Fang. Integrated optimization scheme of mechanical and processing sequence method. Fixture design and manufacture of, 2013, 4(3): 34-38.

[3] X.D. Wang, W. Xiao. A comparative study of several evolutionary algorithms to solve multiobjective optimization problems. Computer knowledge and technology, 2013, 4(1): 61-64.

[4] G.D. Liu, Z.F. Zhang. Comparison of an improved particle swarm optimization algorithm and genetic algorithm. Fire and command control, 2013, 4(7): 78-81.

[5] X.R. Li. Particle swarm optimization algorithm. Computer and modernization, 2013, 5(2): 8184.

[6]C.H. Ceng, Z.W. Ning, H.P. Liao. Study on interactive fixture design system based on ADAMS. Manufacturing technology and machine tool, 2012, 5(2): 91-94. 
[7] F.L. Wang, L.P. Dai. Computer aided fixture design system based on ObjectARX. Modern machinery, 2013, 4(5): 54-58.

[8] H.Q. Wan, M.R. Yao, B. Wei, H.G. Hou. Solution, 3D fixture design based on SolidWorks. Manufacturing technology and machine tool, 2012, 4(3): 52-56.

[9] J. Cai, L. Duan, T. Yao, H.J. Xu. Review and development trends of computer aided fixture design. Mechanical design, 2013, 4(1): 65-68.

[10] G.D. Li, H.Q. Wang, C. Li, X.F. Gao, W.X. Li. Simultaneous optimization of weak rigidity workpiece clamping sequence and fixture layout. Engineering and test, 2012, 4(5): 12-15.

[11] Y. Liu, X.Q. Hu. Low rigidity structure part fixture layout optimization technology based on ABAQUS. Tools technology, 2013, 4(4): 24-28.

[12] N. Chen, Z.F. Li, J.Z. Chen. Five kinds of intelligent algorithms to solve the max cut problem. Journal of Naval Aeronautical Engineering Institute, 2012, 4(3): 101-105.

[13] Y.S. Dong, A.N. Zhang. A comparative study on particle swarm optimization algorithm, BP algorithm and genetic algorithm. Information aspect, 2013, 6(5): 338-339.

[14] X.Q. You, J.P. Qiu, M. Lin, G.S. Wu, Z.L. Ma. A comparative analysis of bionic intelligence algorithms. Fujian computer, 2012, 2(1): 18-21. 